Taking each $r$ equal to unity, we have the interesting special case

$$
X \leqq \max \left\{1+\left|\beta_{1}\right|, 1+\left|\beta_{2}\right|, \cdots, 1+\left|\beta_{n-1}\right|,\left|\beta_{n}\right|\right\} .
$$

Again, it is easy to show also that

$$
X \leqq \max \{|\alpha|+|\beta|,|\beta|+|\gamma|, \cdots,|\lambda|+|\mu|,|\mu|\},
$$

where the quantities $\alpha, \beta, \cdots, \mu$ are defined by the relations

$$
\alpha=\beta_{1}, \beta^{2}=\beta_{2}, \beta \gamma^{2}=\beta_{3}, \beta \gamma \delta^{2}=\beta_{4}, \cdots, \beta \gamma \cdots \lambda \mu^{2}=\beta_{n} .
$$

Through other special choices of the quantities $r$ numerous rather elegant special inequalities may be obtained, several of which are given explicitly by Kojima (loc. cit.).

In case some of the coefficients of equation (1) are zero it may be preferable to employ the second italicized theorem in $\S 6$ and apply to it the principal theorem of this section (or the special cases of the latter).

It is clear that other general formulas may be found for upper bounds to $X$ by employing a sequence of equations each of which is linear or quadratic, such sequence arising by the repeated application of the theorem of this section.

UNIVERSITY OF ILLINOIS, October, 1917.

\title{
THE SOLUTION OF THE WAVE EQUATION BY MEANS OF DEFINITE INTEGRALS.
}

BY PROFESSOR H. BATEMAN.

ThE wave equation

$$
\frac{\partial^{2} V}{\partial x^{2}}+\frac{\partial^{2} V}{\partial y^{2}}+\frac{\partial^{2} V}{\partial z^{2}}=\frac{1}{c^{2}} \frac{\partial^{2} V}{\partial t^{2}}
$$

is the oldest member of the family of partial differential equations, and although he was born without the second and third terms he soon acquired these and played a prominent part in mathematical physics at a time when very few partial differential equations had become famous. With the advent of the mathematical theory of elasticity and Maxwell's electromagnetic theory of light he gained a new lease of life and more 
than doubled his vitality, so that his power is strongly felt even at the present time. In a spirit of reverence for age and valuable service we can still learn much by consulting the old pioneer; not only is he able to throw light on the much discussed subject of the nature of electricity and the general theory of partial differential equations, but he is leaving us a legacy of unsolved problems concerning the diffraction of sound and electric waves and these will tax the ingenuity of mathematicians for years to come. It is often said that Maxwell's equations do not tell us anything about the structure of the æther, but this view may not be correct. Although the equations themselves do not give any clear indications of the properties of the æther, yet the solutions of the equations may give some information. It must be realized that when a physical problem is reduced to differential equations some of the features of the problem are eliminated in the process and very often these features are of great physical interest. Unfortunately we cannot retrace the process of elimination and the only process of reconstruction open to us is to make an exhaustive study of the solutions of the differential equations and to pay particular attention to cases in which a very general solution is built up from elementary solutions of a particularly simple type.

In the solution of diffraction problems the modern tendency seems to be to make use of integrals taken along complex paths in representing the required solutions of the wave equation.* This method is very powerful and beautiful, but to obtain a visualization of the physical processes which actually take place it seems desirable to make use of integrals taken along real paths and some progress has already been made in this direction. Again in A. W. Conway's derivation $\dagger$ of the potentials for a moving point charge of electricity use is made of contour integrals of type

$$
\int \frac{f(\tau) d \tau}{[x-\xi(\tau)]^{2}+[y-\eta(\tau)]^{2}+[z-\zeta(\tau)]^{2}-c^{2}(t-\tau)^{2}}
$$

* See, for instance, A. Sommerfeld, Math. Ann., Bd. 45 (1894), Bd. 47 (1896); Zeitschr. für Math. u. Phys., Bd. 46 (1901); H. M. Macdonald, Electric Waves (1902); Proc. London Math. Soc., ser. 2, vol. 14 (1915), p. 410; H. S. Carslaw, Proc. London Math. Soc., ser. 1, vol. 30 (1898), p. 121; Math. Ann., Bd. 75 (1914), p. 133; T. J. I'A. Bromwich, Proc. London Math. Soc., ser. 2, vol. 14 (1915), p. 450; F. J. W. Whipple, ibid., vol. 16 (1917), p. 94.

$\dagger$ Proc. London Math. Soc., ser. 2, vol. 1 (1903). 
but it has been shown recently* that a similar derivation is possible by taking the integral (2) between certain consecutive real limits $\tau_{1}$ and $\tau_{2}$, each of which is defined by a pair of equations of type

$$
\begin{aligned}
& {[x-\alpha(\theta)]^{2}+[y-\beta(\theta)]^{2}+[z-\gamma(\theta)]^{2}=c^{2}(t-\theta)^{2}, \quad t \geqq \theta,} \\
& \begin{aligned}
{[\alpha(\theta)-\xi(\tau)]^{2}+[\beta(\theta)-\eta(\tau)]^{2}+[\gamma(\theta)} & -\zeta(\tau)]^{2} \\
& =c^{2}(\theta-\tau)^{2}, \quad \theta \geqq \tau .
\end{aligned}
\end{aligned}
$$

It is easy to verify that the definite integral is in this case a wave function; $\dagger$ it is also easy to verify that an integral of the above type represents a wave function when the limits are functions of type $\tau$ which is defined by an equation such as $l(\tau)[x-\xi(\tau)]+m(\tau)[y-\eta(\tau)]+n(\tau)[z-\zeta(\tau)]=c(t-\tau)$, where

$$
[l(\tau)]^{2}+[m(\tau)]^{2}+[n(\tau)]^{2} \equiv 1 .
$$

This brings us to the consideration of the following problem: If $f(x, y, z, t, \theta)$ is a wave function for all values of the parameter $\theta$, the definite integral

$$
V=\int_{\theta_{1}}^{\theta_{2}} f(x, y, z, t, \theta) d \theta
$$

is certainly a wave function when $\theta_{1}$ and $\theta_{2}$ are suitable constants, but it may also be a wave function when $\theta_{1}$ and $\theta_{2}$ are certain functions of $x, y, z$ and $t$. The problem is to find a suitable pair of functions $\theta_{1}$ and $\theta_{2}$ when $f(x, y, z, t, \theta)$ is given.

Let us commence by considering the case when $f$ is a wave function of type $\gamma F(\alpha, \beta)$ where $F$ is an arbitrary function of its two arguments and $\alpha, \beta, \gamma$ are functions of $x, y, t$ and $z$. This type of wave function may be regarded as more fundamental even than the familiar function

$$
\left[(x-\xi)^{2}+(y-\eta)^{2}+(z-\zeta)^{2}-c^{2}(t-\tau)^{2}\right]^{-1},
$$

* H. Bateman, Tohôku Math. Journal, vol. 13.

$\dagger$ The analogous solution of the wave equation in two dimensions may be used to solve the problem of the diffraction by a half plane of the waves from a moving line source of sound, the line being parallel to the edge of the diffracting plane.

$\ddagger$ The problem of finding all possible forms for $\alpha, \beta$ and $\gamma$ is solved in this BulletiN, vol. 21 (1915), p. 299, and in Amer. Journal of Mathematics, April, 1915. We may write

$$
\begin{aligned}
& z-c t=\phi(\alpha, \beta)+(x+i y) \chi(\alpha, \beta), \\
& z+c t=\psi(\alpha, \beta)-(x-i y) / \chi(\alpha, \beta) .
\end{aligned}
$$


which gives rise to the integrand in (2). In electromagnetic theory, for instance, if $E$ and $H$ denote electric and magnetic intensities respectively, the æthereal field in which the complex vector $M \equiv H+i E$ is represented by the expressions

$$
M=F(\alpha, \beta)(\nabla \alpha \times \nabla \beta) \equiv \frac{i}{c} F(\alpha, \beta)\left[\frac{\partial \beta}{\partial t} \nabla \alpha-\frac{\partial \alpha}{\partial t} \nabla \beta\right]
$$

is a pure radiant field, while the quantities $\alpha$ and $\beta$ are constant along the rays which are the rectilinear paths of the energy. It has been shown that the field of a point charge of electricity can be regarded as the limiting case of two superposed radiant fields of the above description in each of which electricity flows with the velocity of light along certain pairs of singular rays. The singular rays in the two fields overlap and there is a cancelling of electricity except on two segments which shrink to a point as the limit is approached. This suggests that the æther may consist of particles of electricity and electric doublets travelling along straight lines with the velocity of light. In this connection it may be worth while to point out that the rays are bicharacteristics* of the partial differential equations while $\alpha$ and $\beta$ are functions which satisfy the partial differential equation of the characteristics of equation (1).

Let us now make $\alpha$ and $\beta$ depend on a parameter $\theta$ as well as on $x, y, t$ and $z$; forming the definite integral

$$
\begin{aligned}
M=\int_{\theta_{1}}^{\theta_{2}} F(\alpha, \beta, \theta)(\nabla & \times \nabla \beta) d \theta \\
& \equiv \frac{i}{c} \int_{\theta_{1}}^{\theta_{2}} F(\alpha, \beta, \theta)\left(\frac{\partial \beta}{\partial t} \nabla \alpha-\frac{\partial \alpha}{\partial t} \nabla \beta\right) d \theta
\end{aligned}
$$

and writing

$$
\frac{\partial \alpha}{\partial x} \frac{\partial \theta}{\partial x}+\frac{\partial \alpha}{\partial y} \frac{\partial \theta}{\partial y}+\frac{\partial \alpha}{\partial z} \frac{\partial \theta}{\partial z}-\frac{1}{c^{2}} \frac{\partial \alpha}{\partial t} \frac{\partial \theta}{\partial t} \equiv(\alpha, \theta),
$$

we find that

$$
\operatorname{rot} M+\left.\frac{i}{c} \frac{\partial M}{\partial t} \equiv\{(\beta, \theta) \nabla \alpha-(\alpha, \theta) \nabla \beta\} F(\alpha, \beta, \theta)\right|_{\substack{\theta=\theta_{2} \\ \theta=\theta_{1}}} .
$$

The right-hand side is clearly zero if $\theta_{1}$ and $\theta_{2}$ are solutions of

* The theory of bicharacteristics is expounded in Hadamard's Propagation des Ondes. 
the two equations

$$
(\alpha, \theta)=0 \text { and }(\beta, \theta)=0 .
$$

Now $(\alpha, \alpha)=0,(\alpha, \beta)=0$ and $(\beta, \beta)=0$, hence the above equations may be satisfied by taking $\theta_{1}$ and $\theta_{2}$ to be roots of the equations

$$
G_{1}(\alpha, \beta, \theta)=0 \text { and } G_{2}(\alpha, \beta, \theta)=0
$$

respectively. ${ }^{*}$ It should be noticed in passing that $\theta_{1}$ and $\theta_{2}$ are solutions of the characteristic equation $(\theta, \theta)=0$. Since

$$
\operatorname{rot} M+\frac{i}{c} \frac{\partial M}{\partial t}=0
$$

it follows that each component of $M$ satisfies the wave equation and so we have an important class of wave functions represented by definite integrals in which the limits are functions of $x, y, z$ and $t$. It should be noticed that if we substitute a definite integral of type

$$
V=\int_{\theta_{1}}^{\theta_{2}} \gamma F(\alpha, \beta, \theta) d \theta
$$

in the wave equation we obtain a system of partial differential equations for $\theta_{1}$ and $\theta_{2}$ and these are difficult to solve directly. The above method, however, indicates the nature of the complete solution of these equations. It also emphasizes the advantage which is gained by using a system of partial differential equations of the first order instead of the second order equation (1). This plan might be adopted with advantage in other cases; it may be recalled also that the first order equations may be used more profitably than (1) in the determination of all the functions of type $\alpha$ and $\beta$.

The theorem that has just been proved is of wide application. We know for instance that a wave function of type $\gamma F(\alpha, \beta)$ may be obtained by writing

$$
\gamma=\frac{1}{R}, \quad \alpha=t-\frac{R}{c}, \quad \beta=\frac{x-i y}{R+z},
$$

where $R$ is the distance from the origin. We may generalize this solution by shifting the origin to the point $\xi(\theta), \eta(\theta)$,

\footnotetext{
${ }^{*} G_{1}$ and $G_{2}$ are arbitrary functions of $\alpha, \beta$ and $\theta$.
} 
$\zeta(\theta)$ and we then have the result that if

$$
\begin{gathered}
r^{2}=[x-\xi(\theta)]^{2}+[y-\eta(\theta)]^{2}+[z-\zeta(\theta)]^{2}, \\
\alpha=t-\frac{r}{c}, \quad \beta=\frac{x-\xi(\theta)-i[y-\eta(\theta)]}{r+[z-\zeta(\theta)]},
\end{gathered}
$$

the definite integral

$$
V=\int_{\theta_{1}}^{\theta_{2}} F(\alpha, \beta, \theta) \frac{d \theta}{r}
$$

satisfies the wave equation, provided $\theta_{1}$ and $\theta_{2}$ are roots of the equations

$$
G_{1}(\alpha, \beta, \theta)=0 \text { and } G_{2}(\alpha, \beta, \theta)=0
$$

respectively. The case in which $F, G_{1}$ and $G_{2}$ are independent of $\beta$ is of special interest. $V$ may then be regarded as the velocity potential of a chain of sources of sound each of which is only active for a certain interval of time which may be different for different sources.

Throop College of Technology, Pasadena, October 6, 1917.

\section{LITERATURE OF PURE MATHEMATICS.}

Historical Introduction to Mathematical Literature. By G. A. Miller. New York, Macmillan, 1916. $14+302$ pp. Price $\$ 1.60$.

IT is difficult to overestimate the extent of inspiration which may emanate from interesting exposition of problems and wonders of science in a form intelligible to those who are not deeply versed in the subject. How much richer must be the intellectual outlook of thousands throughout the world who have read: Science and Hypothesis, The Value of Science, and Science and Method! Is it hard to believe that the future historian may some day tell us that the very notable stage of advancement of astronomy in America in 1910 was not a little due to Simon Newcomb's remarkable gifts of popularization of his subject, exercised through written and spoken word in magazine, book, society, and congress during 